\title{
Defining the Cause of Postpartum Myocardial Infarction - Another Use for Optical Coherence Tomography
}

\author{
Abdullah Alenezi ${ }^{1}$, Jadan Alsaddah ${ }^{1}$, Ossama Maadarani ${ }^{2}$. \\ ${ }^{1}$ Adult Cardiology Department, Chest Diseases Hospital, Kuwait \\ ${ }^{2}$ Internal Medicine Department, Ahmadi Hospital - Kuwait Oil Company, Kuwait
}

Received: 30/06/2020

Accepted: $13 / 07 / 2020$

Published: $17 / 08 / 2020$

\begin{abstract}
How to cite this article: Alenezi A, Alsaddah J, Maadarani O. Defining the cause of postpartum myocardial infarction - another use for optical coherence
\end{abstract} tomography. EJCRIM 2020;7: doi:10.12890/2020_001854.

Conflicts of Interests: The Authors declare that there are no competing interests.

This article is licensed under a Commons Attribution Non-Commercial 4.0 License

\section{ABSTRACT}

Chest pain in a young postpartum female can have many causes; however, when associated with ST elevation on ECG, spontaneous coronary artery dissection (SCAD) should be high on the list. Coronary angiography remains the first step in delineating the coronary lesion in suspected cases of SCAD and optical coherence tomography (OCT) can be crucial when the angiographic appearance remains uncertain. We present a case of a young postpartum female with ST elevation myocardial infarction (STEMI). Coronary angiography revealed a dilated part of the middle segment of the left anterior descending (LAD) artery while intramural haematoma (IMH) of the coronary artery wall was found on OCT, which confirmed the clinically suspected diagnosis of SCAD.

\section{LEARNING POINTS}

- $\quad$ SCAD is a rare entity and considered the most common cause of STEMI in the postpartum state.

- OCT is an intracoronary imaging modality utilizing light waves for image acquisition with high resolution, assisting in clinical decisionmaking regarding the pathophysiological mechanism of an acute coronary syndrome (ACS).

- In suspected cases of SCAD, coronary angiography remains the first diagnostic line and OCT can be crucial in confirming an ambiguous angiographic appearance.

\section{KEYWORDS}

Spontaneous coronary artery dissection, optical coherence tomography, postpartum

\section{INTRODUCTION}

Pregnancy-associated myocardial infarction (MI) is non-atherosclerotic in most cases and spontaneous coronary artery dissection (SCAD) is considered the main reason for this entity. Dissection of an epicardial coronary vessel with no obvious link to cardiac catheterization, trauma or atherosclerosis defines SCAD [1]. In SCAD, the mechanism of coronary obstruction occurs due to intramural haematoma (IMH) of the arterial wall or an intimal tear, and due to the limitations of conventional coronary angiography the angiographic appearance can be uncertain. Optical coherence tomography (OCT) is an intracoronary imaging modality that provides great detail on the lumen and wall of the coronary vessel and helps in clinical decision-making, especially when the angiographic appearance is uncertain.

We present a case of a 26-year-old woman with ST elevation myocardial infarction (STEMI) 10 days postpartum. The angiographic appearance during coronary angiography was not typical of SCAD; however, due to the paucity of atherosclerotic risk factors in a young postpartum female presenting with STEMI, SCAD was highly suspected and the use of OCT was crucial in confirming IMH of the coronary artery wall. 


\section{CASE DESCRIPTION}

A 26-year-old female, who had an uneventful full-term vaginal delivery of a healthy baby 10 days previously, presented to the emergency department (ED) with recurrent episodes of chest pain, mainly at rest, with intermittent radiation to her back starting on the fifth day after hospital discharge. The frequency of these attacks peaked on the day of admission. There was no history of atherosclerotic risk factors and she did not smoke. She denied alcohol consumption or use of recreational drugs. She had 1 normal delivery previously and no abortions. There was no family history of sudden cardiac death, congenital or acquired cardiac disease or connective tissue disease. In the ED she had mild chest pain but was not in distress. Examination revealed blood pressure of 110/70 mmHg, heart rate of 85 beats/minute, respiratory rate of 18 breaths/minute and oxygen saturation of $98 \%$ on room air. The chest and cardiac examinations were unremarkable and there was no lower limb swelling. The first electrocardiogram (ECG) showed ST segment elevation in the antero-inferior leads with a QS pattern in most of the precordial leads (Fig. 1). The high-sensitivity cardiac troponin T test (hs-cTnT) showed 27,000 ng/l (normal $<15 \mathrm{ng} / \mathrm{l}$ ). Echocardiography showed mild hypokinesia of the anteroseptal wall and inferior wall. After a loading dose of aspirin and clopidogrel, the patient was transferred from the ED to the catheterization laboratory for coronary angiography, which showed a dilated part of the middle segment of the left anterior descending (LAD) artery with TIMI III flow and intact left circumflex and right coronary arteries (Fig. 2).

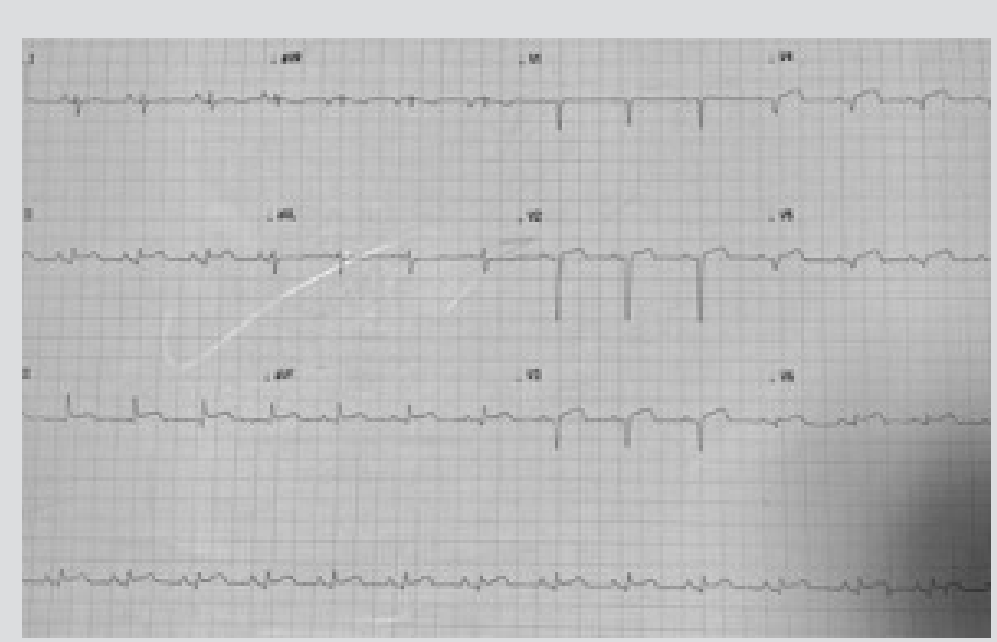

Figure 1. ST elevation in the inferior leads and precordial leads with a QS pattern

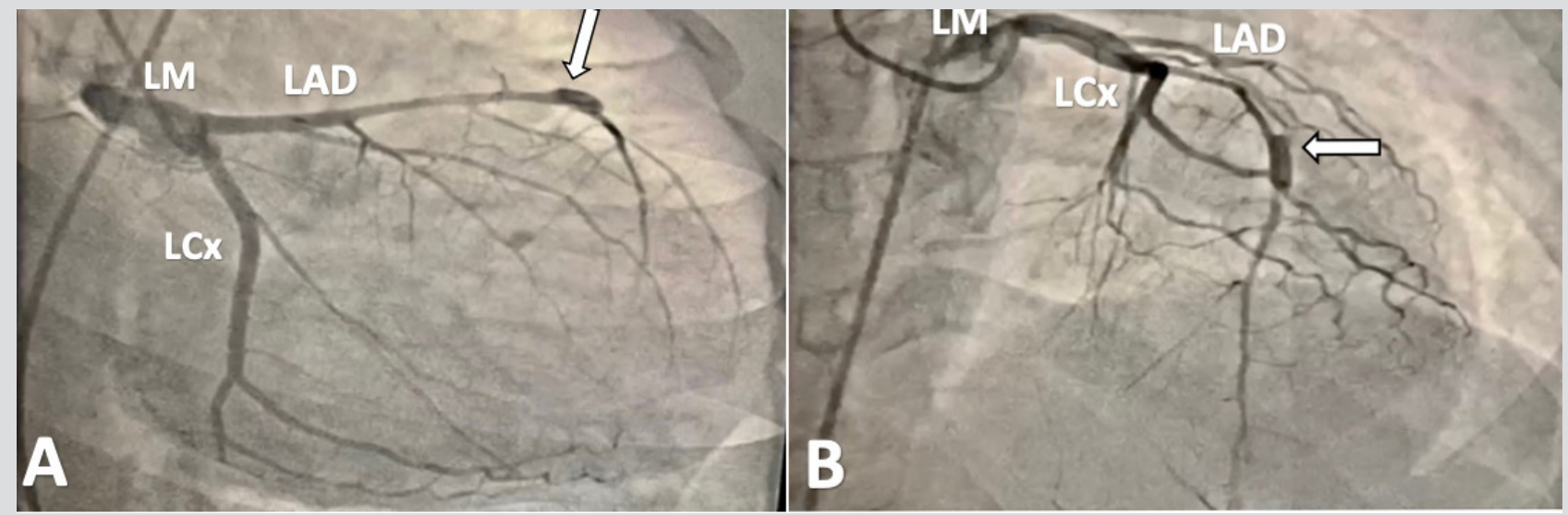

Figure 2. Coronary angiography in different angiographic views; view A and view B. In both views a dilated part of the middle segment of the left anterior descending (LAD) artery was seen (white arrows). LCx: Left circumflex artery; LM: left main coronary artery

The angiographic picture was not consistent with the typical angiographic appearance of SCAD. Due to the demographic characteristics of the patient and her postpartum state, the possibility of SCAD was kept high by the angiographer, and with the use of OCT the suspicion of SCAD was confirmed and IMH compressing the true lumen of the LAD artery was found (Fig. 3). The occurrence of single-vessel disease in addition to the haemodynamic stability of the patient encouraged conservative treatment, and the patient continued on dual antiplatelet therapy in addition to metoprolol with close observation in the intensive care unit. During her stay in the hospital, the patient had no 
recurrence of chest pain and haemodynamics remained stable. The patient was discharged home on Day 8 . On follow-up after 2 months she was fine, with no recurrence of chest pain.

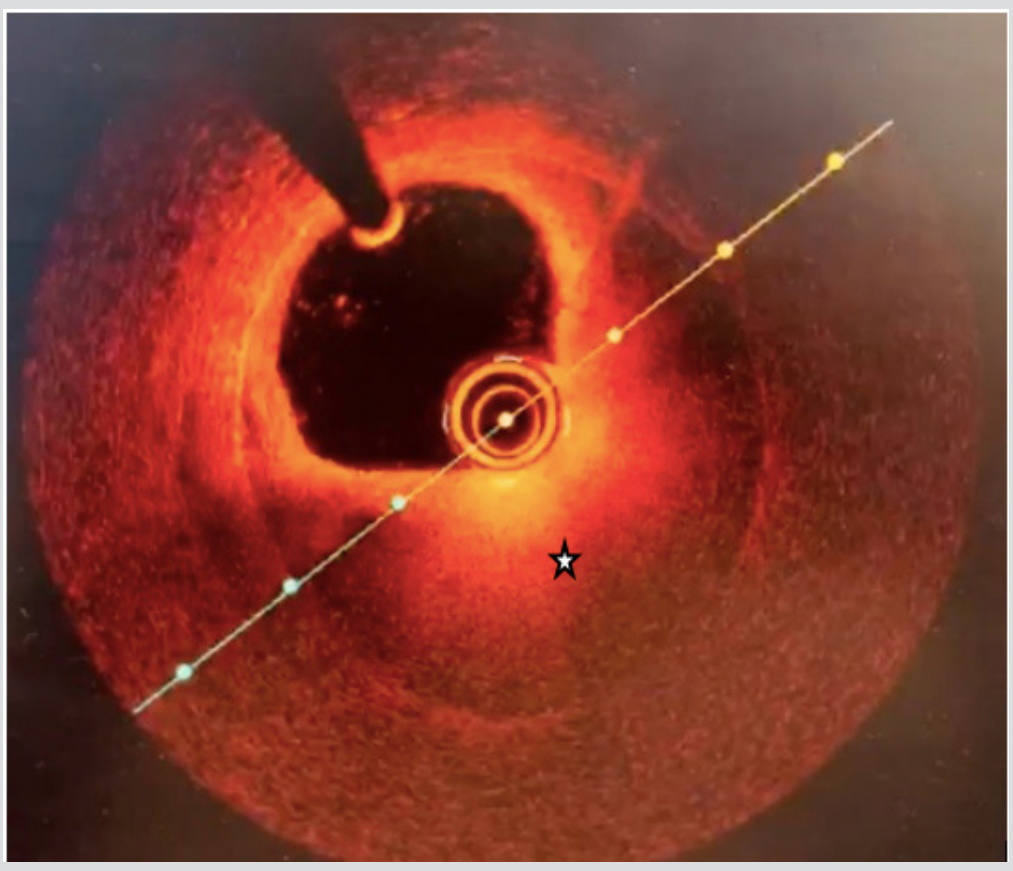

Figure 3. Optical coherence tomography showing intramural haematoma (star)

\section{DISCUSSION}

Pregnancy-associated MI is mostly caused by SCAD ${ }^{[1]}$, which is defined as the dissection of an epicardial coronary vessel with no obvious link to cardiac catheterization, trauma or atherosclerosis ${ }^{[2]}$. The compromise in coronary flow occurring during coronary artery dissection develops either from spontaneous rupture of microvessels inside the coronary artery wall forming IMH or an intimal tear generating a false lumen compressing the true lumen of the coronary artery and resulting in acute MI. The prevalence of SCAD in the general population as an unusual cause of acute coronary syndrome (ACS) ranges from $0.07-1 \%{ }^{[3]}$ and peaks at $43 \%$ in pregnancy-associated MI ${ }^{[2]}$. Almost 5 decades passed from the first description of SCAD on autopsy in 1931 of a young female who died suddenly to the first angiographic description for a 55-year-old female with acute $\mathrm{MI}^{[4]}$.

OCT is an intravascular imaging technique that differs from intravascular ultrasound (IVUS) in utilizing light waves in the near-infrared range instead of sound waves to obtain cross-sectional images of the coronary artery with 10-fold higher image resolution compared to IVUS. The high image resolution provides great detail of both the coronary artery lumen and wall, which allows discrimination of the underlying mechanism of ACS and assists in clinical decision-making with better delineation of angiographic uncertainty ${ }^{[5]}$. The first documented use of OCT as an advanced intracoronary imaging technique was in $2008{ }^{[6]}$. Due to the lack of randomized experimental data supporting a prognostic role for OCT in catheter-based intervention, its clinical applications are not yet fully established. However, there is expert consensus that OCT can provide valuable information on the extent of coronary atherosclerosis, the degree of luminal narrowing, the possibility of vascular injury post-stent implantation as well as incomplete stent apposition.

The most vulnerable periods for pregnancy-associated SCAD are the third trimester and postpartum, although SCAD has been reported in the early weeks of gestation and in the late postpartum period ${ }^{[7]}$. Hormonal changes during the peripartum period and their effects on the coronary receptors probably play an important role in weakening the coronary wall architecture and subsequent intimal rupture and occurrence of IMH; however, the cause of pregnancy-associated SCAD is still not clearly understood ${ }^{[2]}$.

The whole spectrum of ACS can be the presenting picture of SCAD where 26 to $87 \%$ of patients present with STEMI. Cardiogenic shock, ventricular arrhythmias or sudden cardiac death can be a manifestation of SCAD ${ }^{[8]}$. Despite the limitation of conventional coronary angiography being lumenography and not imaging the arterial wall specifically, it remains the first-line diagnostic imaging modality in suspected cases of SCAD. Three types of angiographic appearance are recognized in patients with SCAD. Type 1 refers to the presence of multiple radiolucent lumens and is considered pathognomonic although it appears in a small proportion of cases ${ }^{[9]}$. A diffuse smooth narrowing with length usually $>2 \mathrm{~cm}$ is consistent with type 2 and considered the most common angiographic manifestation of SCAD [10] 
Focal or tubular (short) stenosis that mimics atherosclerosis is seen much less frequently and is consistent with type 3 . Keeping in mind the differences in the angiographic appearance of this rare entity, angiographers should not solely rely on the classic angiographic picture of wall contrast staining because of the risk of missing up to 70\% of SCADs: this should encourage the use of intracoronary imaging techniques to clarify a subtle or ambiguous appearance to demonstrate IMH and a double lumen.

An individualized approach should be taken for patients with SCAD based on haemodynamic stability, the site of dissection, the number of vessels involved and the presence of ongoing ischaemia. A conservative approach is considered the best choice if haemodynamic parameters are stable, there is single-vessel disease with a distal lesion site and there is no evidence of ongoing ischaemia; this includes antiplatelet agents (single or dual), beta blockers and nitrates ${ }^{[11]}$. Urgent intervention with percutaneous coronary intervention or coronary artery bypass grafting (CABG) should be considered in high-risk patients with haemodynamic instability, a left main lesion or ongoing ischaemia. In our case, the presentation of a young postpartum female with STEMI raised the possibility of SCAD, where the angiographic uncertainty arising during coronary angiography necessitated the advantageous use of OCT to delineate the ambiguous appearance and discriminate the pathophysiological mechanism underlying STEMI. Conservative therapy was chosen since the patient had single-vessel disease and her haemodynamics were stable, and was successful.

\section{CONCLUSION}

SCAD is a rare entity and considered the most common cause of STEMI in the postpartum state, while coronary angiography remains the first diagnostic line. OCT is an intracoronary imaging modality that plays a crucial role in delineating an ambiguous angiographic appearance and confirming a clinically suspected diagnosis.

\section{REFERENCES}

1. Elkayam U, Jalnapurkar S, Barakkat MN, Khatri N, Kealey AJ, Mehra A, et al. Pregnancy-associated acute myocardial infarction: a review of contemporary experience in 150 cases between 2006 and 2011. Circulation 2014;129:1695-1702.

2. Hayes SN, Kim ESH, Saw J, Adlam D, Arslanian-Engoren C, Economy KE, et al. Spontaneous coronary artery dissection: current state of the science: a scientific statement from the American Heart Association. Circulation 2018;137(19):e523-e557.

3. Vanzetto G, Berger-Coz E, Barone-Rochette G, Chavanon O, Bouvaist H, Hacini R, et al. Prevalence, therapeutic management and medium-term prognosis of spontaneous coronary artery dissection: results from a database of 11,605 patients. Eur J Cardiothorac Surg 2009;35:250-254.

4. Ciraulo DA, Chesne RB. Coronary arterial dissection: an unrecognized cause of myocardial infarction, with subsequent coronary arterial patency. Chest 1978;73:677-679.

5. Guagliumi G, Capodanno D, Saia F, Musumeci G, Tarantini G, Garbo R, et al. Mechanisms of atherothrombosis and vascular response to primary percutaneous coronary intervention in women versus men with acute myocardial infarction: results of the OCTAVIA study. JACC Cardiovasc Interv 2014;7:958-968.

6. Alfonso F, Canales E, Aleong G. Spontaneous coronary artery dissection: diagnosis by optical coherence tomography. Eur Heart J 2009;30:385.

7. Vijayaraghavan R, Verma S, Gupta N, Saw J. Pregnancy-related spontaneous coronary artery dissection. Circulation 2014;130:1915-1920.

8. Nakashima T, Noguchi T, Haruta S, Yamamoto Y, Oshima S, Nakao K, et al. Prognostic impact of spontaneous coronary artery dissection in young female patients with acute myocardial infarction: a report from the Angina Pectoris-Myocardial Infarction Multicenter Investigators in Japan. Int J Cardiol 2016;207:341-348.

9. Saw J. Coronary angiogram classification of spontaneous coronary artery dissection. Catheter Cardiovasc Interv 2014;84:1115-1122.

10. Alfonso F, Paulo M, Dutary J. Endovascular imaging of angiographically invisible spontaneous coronary artery dissection. JACC Cardiovasc Interv 2012;5:452-453.

11. Tweet MS, Eleid MF, Best PJ, Lennon RJ, Lerman A, Rihal CS, et al. Spontaneous coronary artery dissection: revascularization versus conservative therapy. Circ Cardiovasc Interv 2014;7:777-786. 